\title{
Diversity, traditional uses and conservation status of Zingiberaceae in Udorn Thani Province, Thailand
}

\author{
PIYAPORN SAENSOUK ${ }^{1}$, SURAPON SAENSOUK ${ }^{2, v}$ \\ ${ }^{1}$ Plant and Invertebrate Taxonomy and Its Applications Unit Group, Department of Biology, Faculty of Science, Mahasarakham University, Maha \\ Sarakham 44150, Thailand \\ 2Plant and Invertebrate Taxonomy and Its Applications Unit Group, Walai Rukhavej Botanical Research Institute, Mahasarakham University, \\ Kantarawichai District, Maha Sarakham, 44150, Thailand. "email: surapon.s@msu.ac.th
}

Manuscript received: 11 May 2021. Revision accepted: xxx July 2021.

\begin{abstract}
Saensouk P, Saensouk S. 2021. Diversity, traditional uses and conservation status of Zingiberaceae in Udorn Thani Province, Thailand. Biodiversitas 22: 3083-3097. Southeast Asia is recognized as the center of Zingiberaceae distribution with Thailand is among the important regions. Nonetheless, a comprehensive study in a regional context that investigates the biological aspects of the family is lacking. This study aimed to determine the diversity, distribution, ecology, conservation status, and traditional uses of the family Zingiberaceae in Udorn Thani Province, northeastern Thailand. In total, three tribes, nine genera, 47 species of Zingiberaceae were identified during a botanical survey between January and December 2020 in Udorn Thani. Curcuma and Kempferia were the most diverse genera with nine and eight species, respectively, followed by Zingiber as the third most diverse genus with seven species and Alpinia as the fourth most diverse genus with six species. While the genera Etlingera and Hedychium were the least diverse with each containing just one species. For all Zingiberaceae species, the flowering period was found between March and September, while the fruiting period was found between May and October. Zingiberaceae was found with the greatest frequency in five ecosystem types, namely cultivated areas, deciduous dipterocarp forest, mixed deciduous forest, dry evergreen forest and river basin. Twenty-one species were reported as rare species in research area, i.e. Boesenbergia baimaii, B. isanensis, Kaempferia picheansoonthonii, and K. udonensis. It was revealed that the traditional uses of many Zingiberaceae species from villagers in Udorn Thani Province were most frequently used for medicine, food, ornamentals, rituals, spices, perfume and cosmetics. Rhizomes, roots, pseudostems, young inflorescences, inflorescences, young leaves, leaves and fruits were the parts of this plant used.
\end{abstract}

Keywords: conservation of status, diversity, Udorn Thani Province, uses, Zingiberaceae

\section{INTRODUCTION}

Zingiberaceae (Ginger family) is a large family in monocotyledons under the order Zingiberales with about 50 genera of 1,600 species worldwide (Leong-Škorničková et al. 2019). It is widely distributed in the tropical zone throughout tropical Africa, Asia and the Americas. The center of diversity of the family Zingiberaceae is located in Southeast Asia. The dominant characteristic of Zingiberaceae is the unique smell produced in all parts of the plant, especially in the rhizome. The ginger family is used for a broad range of purposes including for food, spices, medicine, dyes, cosmetics, perfume and ornamental plants. Among the large member of Zingiberaceae family, the popular species include bitter ginger (Zingiber zerumbet), galangal (Alpinia galanga), ginger (Zingiber officinale), patumma or Siam Tulip (Curcuma alismatifolia), and turmeric (Curcuma longa) (Chumroenphat et al. 2019).

There were several botanists have studied the diversity and utilizations of Zingiberaceae. For example, Saensouk and Jenjittikul (2001) reported the traditional use of this family in Thailand as a vegetable obtained from the young leaves of Kaempferia grandifolia. Saensouk and Saensouk (2014) also reported a traditional use as a vegetable from young leaves of Elettariopsis biphylla. Koga et al. (2016) reported traditional uses of Zingiber zerumbet for medicinal plants. Pholhiamhan et al. (2018) found 20 species in family Zingiberaceae were used in the daily life of the Phu Thai ethnic group in Nakhon Phanom Province, Thailand. Furthermore, Saensouk and Saensouk (2019) recognized a new species from Northeastern Thailand (i.e., Kaempferia mahasarakhamensis) that has traditional uses. Yob et al. (2011) reported ethnomedicinal, chemical and pharmacological uses of Zingiber zerumbet (L.) Smith. Ayati et al. (2019) reported ethnobotany and traditional uses of Curcuma longa and C. zedoaria. Phumthum and Balslev (2020) reported and identified species from Zingiberaceae family with pharmacological properties using the ICPC-2 Standard. Wahidah et al. (2020) studied the ethnobotany of Zingiberaceae as traditional medicine utilized by the Colo Muria mountain villagers, Central Java, Indonesia. Pham et al. (2021) reported the ethnomedicinal, phytochemistry and pharmacology uses of plant species belonging to Kaempferia genus. Chumroenphat et al. (2021) studied changes in curcuminoids and chemical components of turmeric (Curcuma longa L.) under freeze-drying and lowtemperature drying methods. More recently, Saensouk et al. (2021) published six new species and a new record including traditional uses of Curcuma from Thailand. 
While there are a large diversity and uses of Zingiberaceae, especially in Southeast Asia, several species are to have conservation concern. IUCN (2021) assigned the conservation status of the family Zingiberaceae, i.e., least concern or LC (seven species-Amomum schmidtii, A. repoense, A. villosum var. xantoides, A. trilobum, Boesenbergia rotunda, Curcuma alismatifolia and Zingiber thorelii), data deficient or DD (one species-Amomum uliginosum) and endangered or E (two species, i.e., Globba laeta and G. siamensis). Moreover, several botanists have reported many endemic species, such as Boesenbergia baimaii (Sensouk and Larsen 2001), B. isanensis (Saensouk \& Saensouk 2020), Kaempferia picheansoonthonii (Phokham et al. 2013), and K. udonensis (Phokham et al. 2013).

Many countries are found high diversity of family Zingiberaceae i.e. Thailand is recognized as one of the centers of distribution of Zingiberaceae family with about 26 genera out of 300 species (Saensouk et al. 2016 and Chumroenphat et al. 2019). Udorn Thani Province is located in the northeastern part of Thailand and is the biggest province in the region which includes part of the Phu Phan mountain range. Despite the potentially large number of species from Zingiberaceae family that can be found in the province, a comprehensive study that investigates the biological aspects of the family is lacking. Previously study found that only Saensouk and Saensouk (2020) studied seven genera and 18 species of Zingiberaceae (including a new one to science, i.e., Boesenbergia isanensis) in Phu Phra Bat Historical Park, Ban Phue District, Udorn Thani Province. For this reason, Udorn Thani Province has the highest biodiversity in northeastern Thailand including the large diversity of Zingiberaceae. Therefore, the purpose of this study was to determine the diversity, distribution, ecology, conservation status and traditional uses of the family Zingiberaceae in Udorn Thani Province, northeastern Thailand.

\section{MATERIAL AND METHODS}

\section{Plant materials}

Zingiberaceae specimens were collected from field trips around Udorn Thani Province, northeastern Thailand between January and December 2020 (Figure 1). Voucher specimens obtained from fieldwork were deposited in the Mahasarakham University Herbarium, Thailand. The morphological characteristics of the plant materials were studied under stereo microscopy.

\section{Plant diversity study}

Plant diversity, vernacular names, distribution data and ecological data were taken from the field, herbarium specimens and available literature. The specimens in this study were compared with herbarium specimens that were kept at overseas herbaria i.e. BK: Bangkok Herbarium, Department of Agriculture, Thailand; BKF: The Forest Herbarium, National Parks, Wildlife and Plant Conservation Department, Thailand; KKU: Khon Kaen University Herbarium Thailand; QBG: Queen Sirikit Botanic Garden Herbarium, Thailand., available taxonomic literature or digital images available online. Keys to the tribes, genera and species are provided based on morphological characters.

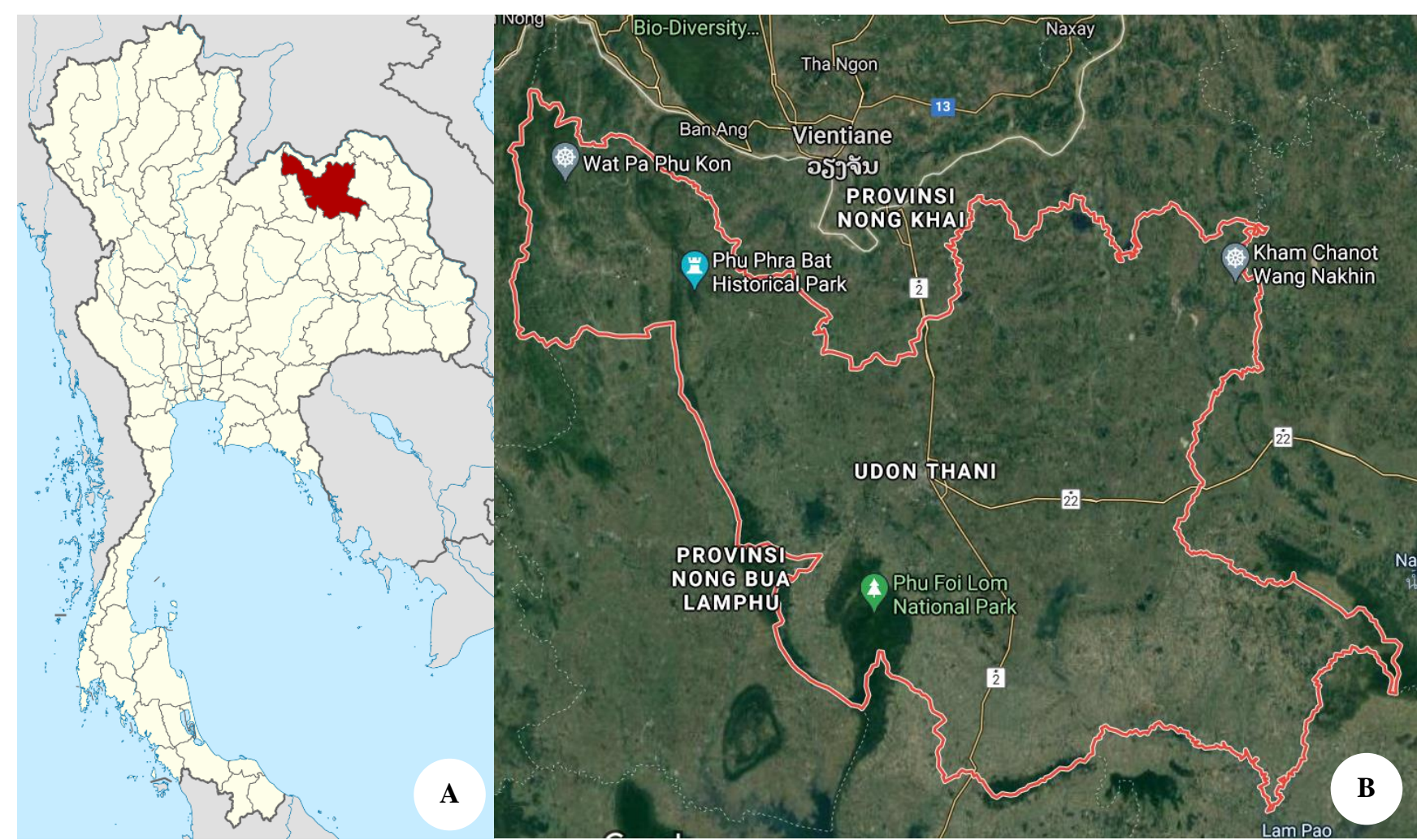

Figure 1. Map of the study location: A. Udorn Thani Province in the context of Thailand; B. Udorn Thani Province in larger detail. (Udorn Thani Province, 2020; www.google.co.th/maps/place/) 


\section{Traditional utilization study}

The traditional data of the Zingiberaceae in Udorn Thani Province were obtained through interviewing 5 local villagers especially folk medicine who lived in this province.

\section{Conservation status study}

The conservation status of the Zingiberaceae is based on information during observations and from collected specimens. In the context of this study, the conservation status can be divided into two categories, namely common species in the research area, and rare species in the research area.

\section{RESULTS AND DISCUSSION}

Zingiberaceae from three tribes, nine genera and 47 species were collected during botanical surveys and collections of specimens from Udorn Thani Province between January and December 2020 (Table 1). All specimens of Zingiberaceae collected from the surveys were successfully identified. The detailed information on the ecological data, distributions, specimens examined, distinguishing features, phenological data, traditional uses, conservation status and keys to tribes, genera and species of Zingiberaceae in Udorn Thani Province are provided in Tables 1 and 2.

\section{Diversity of Zingiberaceae in Udorn Thani Province}

Table 1 shows that the Zingiberaceae recorded in Udorn Thani Province are divided into three tribes based on morphology, namely Alpinieae, Globbeae and Zingibereae. The first tribe is Alpinieae with four genera and 14 species, such as Alpinia (seven species), Amomum (six species) and Etlingera (one species). This tribe can be dived into two groups based on the initiation of the inflorescence. In the first group the inflorescence is exerted from the pseudostem, only Alpinia is recorded in this group. In the second group (Amomum and Etlingera) the inflorescence is exerted from the rhizome. The second tribe is Globbeae, which is comprised of only Globba (five species). The third tribe is Zingibereae with six genera and 27 species that is the largest tribe in this study. The members of tribe Zingibereae are Boesenbergia (three species), Curcuma (nine species), Hedychium (one species), Kaempferia (eight species) and Zingiber (seven species). Keys to the tribes, genera and species are provided based on morphological characteristics and listed below.

\section{Key to tribes of Zingiberaceae in Udorn Thani Province}

1a. Plane of distichy of leaves parallel to rhizome

1b. Plane of distichy of leaves transverse to rhizome

2a. Appendages at anther

2b. No appendages on anther

\section{Key to genera of Zingiberaceae in Udorn Thani Province}

1a. Ovary unilocular with parietal placentation; filament long exerted; Appendaged at anther

Globba

1b. Ovary 3-locular (very rarely unilocular) with central placentation; filament not long exerted; no appendaged at anther

2a. Lateral staminodes reduced to small teeth at base of labellum or wanting, plane of distichy of leaves transverse to rhizome

2b. Lateral staminodes well developed, free from labellum, plane of distichy of leaves pararell to rhizome

3a. Inflorescence terminal on leafy shoot

3b. Inflorescence on separate shoot at base of leafy shoot

4a. Labellum and filament connate into distinct tube above insertion of petals; anther crest absent

4b. Labellum and filament not connate; anther usually crested

5a. Swollen petiole, anther-crest long, enclosing style forming horn-like structure

5b. No swollen petiole, anther-crest variously shaped, but not enclosing style forming horn-like structure

6a. Anther-crest prominent

6b. Anther-crest inconspicuous or absent

7a. Corolla tube long exerted; bract compact and indistinct

7b. Corolla tube short not exerted; bract not compact and distinct

$8 \mathrm{a}$. Inflorescence with coma bracts or without coma bract

8b. Inflorescence without coma bract 
Key to Zingiberaceae species in Udorn Thani Province

\section{Key to Alpinia species}

1a. Bract larger than flower; leaf variegated and bracts green-white

1b. Bract smaller than flower; leaf variegated and bracts green

2a. Leaf margin denticulate

2b. Leaf margin entire

3a. Labellum yellow

3b. Labellum white with red lines

4a. Flower three on cincinnus

4b. Flower one on cincinnus

A. zerumbet

5a. Bracteoles tubular

malaccensis

5b. Bracteoles not tubular

A. siamensis

6a. Labellum with callus-like at base

6b. Labellum without callus-like at base

A. conchigera

A. galanga

\section{Key to Amomum species}

1a. Bracteoles tubular

1b. Bracteoles not tubular

2a. Bract reddish; leaf lower surface reddish

A. wandokthong

2b. Bract greenish; leaf lower surface greenish

A. trilobum

3a. Fruit smooth

3b. Fruit rough with soft spine

4a. All parts glabrescent; fruit many ridged

ides

4b. All parts pubescence; fruit not above

A. repoense

5a. Leaf pubescence

5b. Leaf glabrous

A. schmidtii

A. uliginosum

\section{Key to Globba species}

1a. Inflorescence compact

1b. Inflorescence not compact

2a. Bract white

G. laeta

2b. Bract green

3a. Cincinnus exerted from one bract

G. marantina

3b. Cincinnus not exerted from more than one bract

G. barthei

4a. Bract green

4b. Bract white

G. schomburgii

G. siamensis

\section{Key to Boesenbergia species}

1a. Inside rhizome yellow; labellum orange

1b. Inside rhizome pale yellow; labellum pink or reddish

B. isanensis

2a. Labellum decurved to gound

2b. Labellum recurved from gound

B. baimaii

B. rotunda

\section{Key to Curcuma species}

1a.Inflorescence surrounded by cup-shape involucral bracts with two slits

1b. Inflorescence not surrounded by cup-shape involucral bracts with two slits

2a. Inflorescence without coma bracts

2b. Inflorescence with coma bract

C. campanulata

3a. Rhizome yellow

C. singularis

3b. Rhizome cream or white-cream C. longa

4a. Flower yellow

4b. Flower white with violet

5a. Sheath and leaf sheath red or dark red

5b. Sheath and leaf sheath not as above

C. rubescens

6a. Rhizome branched; blade more than $7 \mathrm{~cm}$ wide

C. comosa

6b. Rhizome unbranched; blade less than $6 \mathrm{~cm}$ wide

. angustifolia

7a. Coma bract shorter than bract

C. parviflora

7b. Coma bract longer than bract

8a. Coma bract pink

8b. Coma bract white 


\section{Key to Kaempferia species}

1a. Inflorescence appears before leaves

1b. Inflorescence appears between leaves

2a. Pseudostem erect from ground

2b. Pseudostem near ground

K. udonensis

3a. Leaf single

3b. Leaf two

5

4a. Lower surface red

4b. Lower surface greenish

K. picheansoonthonii

5a. Pseudostem erect from ground

K. siamensis

5b. Pseudostem near ground

6a. Leaf variegated; all parts of flower purple

K. pulcha

6b. Leaf not variegated; only labellum purple

angustifolia

7a. Leaf margin purple

7b. Leaf margin whitish

$K$. marginata

K. galanga

\section{Key to Zingiber species}

1a. Peduncle on separate shoot at base of leafy shoot, arising oblique with ground

1b. Peduncle on separate shoot at base of leafy shoot, arising vertically to ground

2a. Apex inflorescence tip; labellum cream or pale purple

2b. Apex inflorescence rounded; labellum pale yellow mixed red dots

3a. Labellum pale purple

3b. Labellum cream

4a. Bract brown-dark red

Z. mekongense

Z. rubens

Z. thorelii

Z. monatum

4b. Bract yellow, green or pale yellow when young and red when mature

5

5a. Labellum red

5b. Labellum yellow or cream

Z. officinale

6a. Rhizome pale purple or pale blue

Z. ottensii

6b. Rhizome cream

\section{Phenology}

The phenology, including flowering period and fruiting period of Zingiberaceae in Udorn Thani Province, is presented in Table 1. The flowering period was found to be between March and September, while the fruiting period was found to be between May and October. The genus Alpinia began flowering at two times: March (A. malaccensis, A. mutica and A. zerumbet) and May (four remaining species). The fruiting period began at two times: June (A. malaccensis, A. mutica, and A. zerumbet) and August (four remaining species). Amomum began flowering in May and fruiting in June, but fruiting was not seen in A. wandokthong. The phenology of Etlingera elatior was flowering in March and fruiting in June. Most Globba species started flowering in June except May for $G$. laeta and $G$. siamensis. No Globba species were seen fruiting. Three Boesenbergia species bloomed between June and July and gave fruit in July. Two Curcuma species, $C$. angustifolia and $C$. singularis, bloomed from the rhizome before the pseudostem in March-May and the fruits were present after blooming. Other Сисита species, including $C$. angustifolia, bloomed from the pseudostem in May-August and the fruits were present after blooming. Therefore, $C$. angustifolia bloomed in two periods. The flowers of Hedychium coronarium were present JuneSeptember and the fruits were present after flowering. Two Kaempferia species, $K$. rotunda and $K$. udonensis, bloomed from the rhizome before the pseudostem in March-April and the fruits were present after blooming. Other Kaempferia species bloomed from the pseudostem in May-
August and the fruits were present after blooming. Inflorescences and flowers of Curcuma campanulathus were present in March-May, while the fruits were present in June. All Zingiber species bloomed in July-September and fruited in August-October.

\section{Ecology}

The ecology of zingiberaceous plants is reported in Table 1 . The family was found in five ecosystem types: deciduous dipterocarp forest (containing 19 species), mixed deciduous forest (containing 14 species), dry evergreen forest (containing six species), river basin (only Alpinia conchigera) and cultivated in home gardens (containing 20 species). Boesenbergia baimaii and Kaempferia siamensis were found in new localities. Two endemic species, namely Kaempferia picheansoonthonii and $K$. udonensis, were found only in Udorn Thani Province.

\section{Conservation status}

Table 1 shows the conservation status of family Zingiberaceae in Udorn Thani Province. Common species in the research area were 26 species (comprising Alpinia five species, Amomum one species, Etlingera one species, Globba one species, Boesenbergia one species, Curcuma seven species, Hedychium one species, Kaempferia four species and Zingiber four species). Rare species in the research area were 21 species (comprising Alpinia two species, Amomum five species, Boesenbergia two species, Curcuma two species, Globba three species, Kaempferia two species and Zingiber three species). 
Table 1. Notes on Zingiberaceae species in Udorn Thani Province, Thailand

\begin{tabular}{|c|c|c|c|c|c|c|}
\hline Species & $\begin{array}{l}\text { Specimen } \\
\text { examined }\end{array}$ & Distinguishing features & Distribution & Phenology & Ecology & $\begin{array}{l}\text { Conservation } \\
\text { status }\end{array}$ \\
\hline Alpinia conchigera Griff. & SS. udorn1 & $\begin{array}{l}\text { Bracteoles not tubular, labellum callus- } \\
\text { like }\end{array}$ & $\begin{array}{l}\text { Srangkom, Ban Phue } \\
\text { and Kudjub and Pen } \\
\text { Districts }\end{array}$ & $\begin{array}{l}\text { Fl: May.-Sep. } \\
\text { Fr: Aug.-Oct. }\end{array}$ & $\begin{array}{l}\text { RB, } \\
\text { Cult. }\end{array}$ & $\begin{array}{l}\text { Common species in research } \\
\text { area }\end{array}$ \\
\hline A. galanga (L.) Willd. & SS. udorn2 & $\begin{array}{l}\text { Bracteoles not tubular, labellum not } \\
\text { callus-like }\end{array}$ & All districts & $\begin{array}{l}\text { Fl: May-Sept. } \\
\text { Fr: Aug.-Oct. }\end{array}$ & $\begin{array}{l}\text { DDF, } \\
\text { MDF, } \\
\text { Cult. }\end{array}$ & $\begin{array}{l}\text { Common species in research } \\
\text { area }\end{array}$ \\
\hline A. malaccensis (Burm.f.) Roscoe & SS. udorn3 & One flower per cincinnus & Nayoong District & $\begin{array}{l}\text { Fl: Mar.-June } \\
\text { Fr: June-Oct. }\end{array}$ & $\begin{array}{l}\text { MDF, } \\
\text { DEF }\end{array}$ & Rare species in research area \\
\hline A. mutica Roxb. & SS. udorn4 & Leaf margin denticulate & Ban Phue District & $\begin{array}{l}\text { Fl: Mar.-Aug. } \\
\text { Fr: June-Oct. }\end{array}$ & Cult. & $\begin{array}{l}\text { Common species in research } \\
\text { area }\end{array}$ \\
\hline A. siamensis K.Schum. & SS. udorn5 & $\begin{array}{l}\text { Bracteoles tubular, labellum not callus- } \\
\text { like }\end{array}$ & All districts & $\begin{array}{l}\text { Fl: May-Sept. } \\
\text { Fr: Aug.-Oct. }\end{array}$ & Cult. & $\begin{array}{l}\text { Common species in research } \\
\text { area }\end{array}$ \\
\hline A. vittata W. Bull. & SS. udorn6 & Leaf variegated and bracts green-white & Ban Phue District & $\begin{array}{l}\text { Fl: May-Sept. } \\
\text { Fr: Aug.-Oct. }\end{array}$ & Cult. & $\begin{array}{l}\text { Common species in research } \\
\text { area }\end{array}$ \\
\hline $\begin{array}{l}\text { A. zerumbet (Pers.) B.L.Burtt \& } \\
\text { R.M.Sm. }\end{array}$ & SS. udorn7 & Three flowers per cincinnus & Nayoong District & $\begin{array}{l}\text { Fl: Mar.-May } \\
\text { Fr: June-Oct. }\end{array}$ & MDF & Rare species in research area \\
\hline $\begin{array}{l}\text { Amomum schmidtii (K.Schum.) } \\
\text { Gagnep. }\end{array}$ & SS. udorn8 & Fruits smooth, all parts pubescent & $\begin{array}{l}\text { Ban Phue and Nayoong } \\
\text { Districts }\end{array}$ & $\begin{array}{l}\text { Fl: Mar.-July } \\
\text { Fr: June-Oct. }\end{array}$ & DEF & Rare species in research area \\
\hline A. repoense Pierre ex Gagnep. & SS. udorn9 & Fruits many ridges, all parts glabresence & $\begin{array}{l}\text { Nayoong and Nong } \\
\text { Saeng Districts }\end{array}$ & $\begin{array}{l}\text { Fl: May-July } \\
\text { Fr: Aug.-Sept. }\end{array}$ & DEF & Rare species in research area \\
\hline A. uliginosum J.Koenig & SS. udorn10 & Glabrous in all parts including fruit & $\begin{array}{l}\text { Nayoong and Nong } \\
\text { Saeng Districts }\end{array}$ & $\begin{array}{l}\text { Fl: May-June } \\
\text { Fr: June-Oct. }\end{array}$ & DEF & Rare species in research area \\
\hline $\begin{array}{l}\text { A. villosum var. xantoides (Wall. ex } \\
\text { Baker) T.L.Wu\&S.J.Chen (Figure 2A) }\end{array}$ & SS. udorn11 & Fruit rough with soft spine & Nayoong District & $\begin{array}{l}\text { Fl: May-June } \\
\text { Fr: June-Oct. }\end{array}$ & DEF & Rare species in research area \\
\hline $\begin{array}{l}\text { A. trilobum Gagnep. } \\
\text { (Figure } 2 \mathrm{~B} \text { ) }\end{array}$ & SS. udorn12 & $\begin{array}{l}\text { Bract greenish, leaf lower surface } \\
\text { greenish }\end{array}$ & $\begin{array}{l}\text { Nayoong and Nong } \\
\text { Saeng Districts }\end{array}$ & $\begin{array}{l}\text { Fl: Mar-.Apr. } \\
\text { Fr: June-Oct. }\end{array}$ & MDF & Rare species in research area \\
\hline $\begin{array}{l}\text { A. wandokthong (Picheans. \& } \\
\text { Yupparach) Skornick. \& Hlavatá } \\
\text { (Figure 2C) }\end{array}$ & SS. udorn13 & Bract reddish, leaf lower surface reddish & All districts & $\begin{array}{l}\text { Fl: Mar.-Apr. } \\
\text { Fr: Not seen }\end{array}$ & Cult. & $\begin{array}{l}\text { Common species in research } \\
\text { area }\end{array}$ \\
\hline Etlingera elatior (Jack) R.M.Sm. & SS. udorn14 & Pseudostem upto $4 \mathrm{~m}$ tall, bract red & All districts & $\begin{array}{l}\text { Fl: Mar-May } \\
\text { Fr: June-Sept. }\end{array}$ & Cult. & $\begin{array}{l}\text { Common species in research } \\
\text { area }\end{array}$ \\
\hline Globba barthei Gagnep. & SS. udorn15 & $\begin{array}{l}\text { Inflorescence not compact, cincinnus not } \\
\text { exerted from more than one bract }\end{array}$ & Ban Phue District & $\begin{array}{l}\text { Fl: June-Aug. } \\
\text { Fr: Not seen }\end{array}$ & MDF & Rare species in research area \\
\hline G. laeta K. Larsen & SS. udorn16 & Inflorescence compact, bract white & $\begin{array}{l}\text { Ban Phue and Nayoong } \\
\text { Districts }\end{array}$ & $\begin{array}{l}\text { Fl: May-Aug. } \\
\text { Fr: Not seen }\end{array}$ & DDF & Rare species in research area \\
\hline G. marantina L. & SS. udorn17 & Inflorescence not compact, bract green & $\begin{array}{l}\text { Ban Phue and Nayoong } \\
\text { Districts }\end{array}$ & $\begin{array}{l}\text { Fl: June-July } \\
\text { Fr: Not seen }\end{array}$ & $\begin{array}{l}\text { DDF, } \\
\text { MDF }\end{array}$ & $\begin{array}{l}\text { Common species in research } \\
\text { area }\end{array}$ \\
\hline
\end{tabular}




\section{G. schomburgii Hook.f. \\ G. siamensis (Hemsl.) Hemsl (Figure 2G) \\ Boesenbergia baimaii S.Saensouk \& \\ Larsen (Figure 2D) \\ B. rotunda (L.) Mansf.}

B. isanensis Saensouk \& P. Saensouk (Figure 2E)

Curcuma angustifolia Roxb.

\section{C. alismatifolia Gagnep.}

C. campanulata (Kuntze) Škornick. (Figure 2F)

C. comosa Roxb.

\section{C. longa $\mathrm{L}$.}

C. parviflora Wall.

C. rubescens Roxb.

C. singularis Gagnep.

C. thorellii Gagnep.

Hedychium coronarium J.Koenig

Kaempferia angustifolia Roscoe

K. galanga $\mathrm{L}$.

K. marginata Carey ex Roscoe

K. picheansoonthonii Wongsuwan \& Phokham. (Figure 2H)
SS. udorn18 Inflorescence not compact, cincinnus exerted from one bract, bract green

SS. udorn 19 Inflorescence not compact, cincinnus exerted from one bract, bract white

SS. udorn20 Labellum pink and apex decurved to ground

SS. udorn21 Labellum reddish, apex recurved from ground

SS. udorn22 Inside rhizome yellow, labellum orange

SS. udorn23

Flower yellow, rhizome unbranched, blade less than $6 \mathrm{~cm}$ wide

SS. udorn24 Flower white with violet, coma bract longer than bract, coma bract pink

SS. udorn25 Inflorescence surrounded by cup-shape involucral bracts

SS. udorn26 Flower yellow, rhizome branched, blade more than $7 \mathrm{~cm}$ wide

SS. udorn27 Inflorescence with coma bract, rhizome yellow

SS. udorn28 Flower white with violet, coma bract shorter than bract,coma bract white

SS. udorn29 Flower yellow,

sheath and leaf sheath red or dark red

SS. udorn30 Inflorescence from rhizome without coma bracts

SS. udorn31 Flower white with violet, coma bract longer than bract, coma bract white

SS. udorn32 Flower white

SS. udorn33

Pseudostem with two leaves erect from ground, labellum purple

SS. udorn34 Leaf margin whitish

SS. udorn35 Leaf margin purple

SS. udorn36 Leaf single, lower surface red
Ban Phue and Nayoong

Districts

Ban Phue District

Ban Phue District

All districts

Ban Phue District

All districts

Ban Phue and Kudjub
Districts

Districts

Ban Phue District

All districts

All districts

Ban Phue, Nayoong and

Kudjub Districts

Ban Phue, Nayoong and

Kudjub Districts

All districts

Ban Phue Districts

All districts

Ban Phue District

Ban Phue Districts

Ban Phue Districts

NongWua So and Nong Saeng Districts
Fl: June-Sept. Cult.

Fr: Not seen

Fl: May-Aug. DDF

Fr: Not seen

Fl: June-July

Fr: July

Fl: June-July

Fr: July

Fl: June-July

Fr: July

Fl: Mar-June

July-Sept.

Fr: May-July /

Aug.-Oct.

Fl: July-Aug.

Fr: Aug.-Oct.

DDF,
Cult.

Fl: Mar.-May

Fr: June

Fl: Mar.-Aug.

Fr: May-July

Fl: June-Aug.

Fr: Aug.-Oct.

Fl: May-Aug.

Fr: Aug.-Oct.

Fl: June-Aug.

Fr: Aug.-Oct.

Fl: Mar.-May

Fr: May-June

Fl: June-Aug.

Fr: Aug.-Oct.

Fl: June-Sept

Fr: Sept.-Oct,

Fl: June-July

Fr: July

Fl: June-July

Fr: June-Aug.

Fl: June-Aug.

Fr: July-Aug.

Fl: May-June

Fr: June

DDF,

MDF

MDF

Cult.

DDF,

MDF

DDF

DDF,

MDF

Cult.

DDF

Cult.

Cult.

DDF

DDF
Common species in research area

Rare species in research area

Rare species in research area

Common species in research area

Rare species in research area

Common species in research area

Common species in research area

Common species in research

area

Common species in research area

Common species in research area

area

Common species in research area

Common species in research area

DDF Rare species in research area

Common species in research

area

DDF, Rare species in research area

Common species in research

area

DDF Common species in research area

Rare species in research area 


\begin{tabular}{|c|c|c|c|c|c|c|}
\hline K. pulcha Ridl. & SS. udorn37 & $\begin{array}{l}\text { Pseudostem erect from ground, leaf } \\
\text { variegated, all part of flower purple }\end{array}$ & Ban Phue District & $\begin{array}{l}\text { Fl: June-Aug. } \\
\text { Fr: Not seen }\end{array}$ & Cult. & $\begin{array}{l}\text { Common species in research } \\
\text { area }\end{array}$ \\
\hline K. rotunda $\mathrm{L}$. & SS. udorn38 & $\begin{array}{l}\text { Inflorescence appears before leaves, } \\
\text { pseudostem erect from ground }\end{array}$ & Ban Phue District & $\begin{array}{l}\text { Fl: Mar.-Apr. } \\
\text { Fr: Mar.-May }\end{array}$ & $\begin{array}{l}\text { DDF, } \\
\text { MDF }\end{array}$ & $\begin{array}{l}\text { Common species in research } \\
\text { area }\end{array}$ \\
\hline K. siamensis P.Sirirugsa (Figure 2I) & SS. udorn39 & Leaf single, lower surface greenish & Ban Phue District & $\begin{array}{l}\text { Fl: May-June } \\
\text { Fr: June }\end{array}$ & DDF & Rare species in research area \\
\hline $\begin{array}{l}\text { K. udonensis } \\
\text { Picheans. \& Phokham (Figure 2J) }\end{array}$ & SS. udorn40 & $\begin{array}{l}\text { Inflorescence appears before leaves, } \\
\text { pseudostem near ground }\end{array}$ & $\begin{array}{l}\text { Nong Wua So and Nong } \\
\text { Saeng Districts }\end{array}$ & $\begin{array}{l}\text { Fl: Mar.-Apr. } \\
\text { Fr: Mar.-May }\end{array}$ & DDF & Rare species in research area \\
\hline $\begin{array}{l}\text { Zingiber mekongense Gagnep. (Figure } \\
2 \mathrm{~K})\end{array}$ & SS. udorn41 & $\begin{array}{l}\text { Apex inflorescence rounded, labellum } \\
\text { pale yellow mixed red dots }\end{array}$ & Ban Phue District & $\begin{array}{l}\text { Fl: July-Sept. } \\
\text { Fr: Aug.-Oct. }\end{array}$ & MDF & Rare species in research area \\
\hline $\begin{array}{l}\text { Z. montanum (J.Koenig) Link ex } \\
\text { A.Dietr. }\end{array}$ & SS. udorn42 & $\begin{array}{l}\text { Apex inflorescence tip, bract brown-dark } \\
\text { red }\end{array}$ & All districts & $\begin{array}{l}\text { Fl: July-Sept. } \\
\text { Fr: Aug.-Oct. }\end{array}$ & Cult. & $\begin{array}{l}\text { Common species in research } \\
\text { area }\end{array}$ \\
\hline Z. officinale Roscoe & SS. udorn43 & $\begin{array}{l}\text { Apex inflorescence tip, bract green, } \\
\text { labellum red }\end{array}$ & All districts & $\begin{array}{l}\text { Fl: July-Sept. } \\
\text { Fr: Aug.-Oct. }\end{array}$ & Cult. & $\begin{array}{l}\text { Common species in research } \\
\text { area }\end{array}$ \\
\hline Z. ottensii Valeton & SS. udorn44 & Rhizome pale purple or pale blue & All districts & $\begin{array}{l}\text { Fl: July-Sept. } \\
\text { Fr: Aug.-Oct. }\end{array}$ & Cult. & $\begin{array}{l}\text { Common species in research } \\
\text { area }\end{array}$ \\
\hline Z. rubens Roxb. & SS. udorn45 & Labellum pale purple & Ban Phue District & $\begin{array}{l}\text { Fl: July-Sept. } \\
\text { Fr: Aug.-Oct. }\end{array}$ & MDF & Rare species in research area \\
\hline Z. thorelii Gagnep. (Figure 2L) & SS. udorn 46 & $\begin{array}{l}\text { Apex inflorescence tip, } \\
\text { labellum cream }\end{array}$ & Ban Phue District & $\begin{array}{l}\text { Fl: July-Sept. } \\
\text { Fr: Aug.-Oct. }\end{array}$ & DEF & Rare species in research area \\
\hline Z. zerumbet (L.) Roscoe ex Sm. & SS. udorn47 & $\begin{array}{l}\text { Rhizome cream, bract green when young } \\
\text { and red when mature }\end{array}$ & All districts & $\begin{array}{l}\text { Fl: July-Sept. } \\
\text { Fr: Aug.-Oct. }\end{array}$ & $\begin{array}{l}\text { MDF, } \\
\text { Cult. }\end{array}$ & $\begin{array}{l}\text { Common species in research } \\
\text { area }\end{array}$ \\
\hline
\end{tabular}

Note: DDF: deciduous dipterocarp forest, MDF: mixed deciduous forest, DEF: dry evergreen forest, RB: river basin, Cult.: cultivated, LC: least concern, DD: data deficient, Fl: flowering period, Fr: fruiting period 
Table 2. Traditional uses of Zingiberaceae in Udorn Thani Province, Thailand

\begin{tabular}{|c|c|c|c|c|c|c|c|}
\hline \multirow[t]{2}{*}{ Species } & \multicolumn{7}{|l|}{ Traditional uses } \\
\hline & Food & Spice & Ornamentals & Cosmetics & Perfume & Rituals & Medicines \\
\hline Alpinia conchigera Griff. & $\begin{array}{l}\text { Local people use rhizomes, young } \\
\text { pseudostems and young } \\
\text { inflorescences as food and vegetable }\end{array}$ & $\begin{array}{l}\text { Rhizomes } \\
\text { used as } \\
\text { spice }\end{array}$ & & & & & $\begin{array}{l}\text { Rhizomes used for } \\
\text { stomachache and } \\
\text { skin disease }\end{array}$ \\
\hline A. galanga (L.) Willd. & $\begin{array}{l}\text { Local people use rhizomes, young } \\
\text { pseudostems and young } \\
\text { inflorescences as food and vegetable }\end{array}$ & $\begin{array}{l}\text { Rhizomes } \\
\text { used as } \\
\text { spice }\end{array}$ & & & & & $\begin{array}{l}\text { Rhizomes used for } \\
\text { stomachache and } \\
\text { skin disease }\end{array}$ \\
\hline A. malaccensis (Burm.f.) & Local people use young & & & & & & Rhizomes used for \\
\hline Roscoe & inflorescences as vegetable & & & & & & $\begin{array}{l}\text { stomachache and } \\
\text { skin disease }\end{array}$ \\
\hline A. mutica Roxb. & & & $\begin{array}{l}\text { Whole plant is used as } \\
\text { ornamental }\end{array}$ & & & $\begin{array}{l}\text { Whole plant is used } \\
\text { as power magic }\end{array}$ & \\
\hline A. siamensis K.Schum. & $\begin{array}{l}\text { Local people use rhizomes, young } \\
\text { pseudostems and young } \\
\text { inflorescences as food and vegetable }\end{array}$ & $\begin{array}{l}\text { Rhizomes } \\
\text { used as } \\
\text { spice }\end{array}$ & & & & & $\begin{array}{l}\text { Rhizomes used for } \\
\text { stomachache and } \\
\text { skin disease }\end{array}$ \\
\hline A. vittata W. Bull. & & & $\begin{array}{l}\text { Whole plant used as } \\
\text { ornamental }\end{array}$ & & & & \\
\hline A. zerumbet (Pers.) & Local people use young & & & & & & Rhizomes used for \\
\hline B.L.Burtt \& R.M.Sm. & inflorescences as vegetable & & & & & & $\begin{array}{l}\text { stomachache and } \\
\text { skin disease }\end{array}$ \\
\hline $\begin{array}{l}\text { Amomum schmidtii } \\
\text { (K.Schum.) Gagnep. }\end{array}$ & & & & & & $\begin{array}{l}\text { Whole plant is used } \\
\text { as power magic }\end{array}$ & $\begin{array}{l}\text { Rhizomes used for } \\
\text { stomachache and } \\
\text { skin disease }\end{array}$ \\
\hline A. repoense Pierre ex & & & & & & & Rhizomes and fruits \\
\hline Gagnep. & & & & & & & $\begin{array}{l}\text { used for stomachache } \\
\text { and skin disease }\end{array}$ \\
\hline A. uliginosum J.Koenig & & & & & & & $\begin{array}{l}\text { Rhizomes and fruits } \\
\text { used for stomachache } \\
\text { and skin disease }\end{array}$ \\
\hline $\begin{array}{l}\text { A. villosum var. xantoides } \\
\text { (Wall. ex Baker) T.L.Wu \& }\end{array}$ & & & & & & & $\begin{array}{l}\text { Rhizomes and fruits } \\
\text { used for stomachache }\end{array}$ \\
\hline $\begin{array}{l}\text { S.J.Chen } \\
\text { S.trilobum Gagnen }\end{array}$ & & & & & & & and skin disease \\
\hline A. trilobum Gagnep. & & & & & & & $\begin{array}{l}\text { Rhizomes and fruits } \\
\text { used for stomachache } \\
\text { and skin disease }\end{array}$ \\
\hline $\begin{array}{l}\text { A. wandokthong } \\
\text { (Picheans. \& Yupparach) } \\
\text { Skornick. \& Hlavatá }\end{array}$ & & & & & & $\begin{array}{l}\text { Whole plant is used } \\
\text { as power magic }\end{array}$ & $\begin{array}{l}\text { Rhizomes used for } \\
\text { stomachache and } \\
\text { skin disease }\end{array}$ \\
\hline Skornick. \& Hlavatá & & & & & & & skin disease \\
\hline
\end{tabular}


Etlingera elatior (Jack) R.M.Sm.

Globba barthei Gagnep.

\section{G. laeta K. Larsen}

G. marantina L.

G. schomburgii Hook.f.

G. siamensis (Hemsl.)

Hemsl

Boesenbergia baimaii S.Saensouk \& Larsen

Local people use rhizomes and storage root as food and vegetable

\section{B. rotunda (L.) Mansf.}

Local people use rhizomes and storage root as food and vegetable

B. isanensis Saensouk \& P. Local people use rhizomes and Saensouk

storage root as food and vegetable

\section{Curcuma angustifolia}

Roxb.

C. alismatifolia Gagnep.

Local people use young

inflorescences as food and vegetable

Local people use young

inflorescences as food and vegetable

C. campanulata (Kuntze)

Škornick.

C. comosa Roxb.

C. longa $\mathrm{L}$.

Local people use rhizomes and young inflorescences as food and vegetable
Beautiful inflorescence

cultivated as ornamental

Plants with beautiful

inflorescence cultivated

as ornamental

Plants with beautiful

inflorescence cultivated

as ornamental

Plants with beautiful

inflorescence cultivated

as ornamental

Plants with beautiful

inflorescence cultivated

as ornamental

Plants with beautiful

inflorescence cultivated

as ornamental

Storage

roots are

used as

spice
Rhizomes used for stomachache and

skin disease

Inflorescenceused for

paying respect to the

Buddha

Inflorescenceused for

paying respect to the

Buddha

Inflorescenceused for paying respect to the Buddha

Inflorescenceused for paying respect to the Buddha

Inflorescenceused for

paying respect to the

Buddha

Rhizomes used for stomachache and skin disease

Rhizomes and

storage roots used for stomachache and

skin disease

Rhizomes used for

stomachache and

skin disease

Rhizomes used for

stomachache

Rhizomes used for

stomachache

Rhizomes used for

stomachache and

skin disease

Rhizome used for

stomachache

Local

Rhizomes used for

stomachache 


\begin{tabular}{ll}
\hline C. parviflora Wall. & Local people use young \\
inflorescences as food and vegetable
\end{tabular}

C. rubescens Roxb

C. singularis Gagnep.

C. thorellii Gagnep.

Hedychium coronarium J.Koenig

Kaempferia angustifolia

\section{Roscoe}

K. galanga $\mathrm{L}$.

\section{K. marginata Carey ex} Roscoe

K. picheansoonthonii

Wongsuwan \& Phokham.

K. pulcha Ridl.

\section{K. rotunda $\mathrm{L}$.}

\section{K. siamensis P.Sirirugsa}

K. udonensis

Picheans. \& Phokham

Zingiber mekongense

Gagnep.

Z. montanum (J.Koenig)

Link ex A.Dietr.

Z. officinale Roscoe

\section{Z. ottensii Valeton}

Z. rubens Roxb.

Z. thorelii Gagnep.

Local people use young

inflorescences as food and vegetable

Local people use young

inflorescences as food and vegetable

\section{White flower}

used for

paying respect

to the Buddha

inflorescences as food and vegetable

Local people use young

inflorescences as food and vegetable

Local people use young

inflorescences as food and vegetable

Local people use young

inflorescences as food and vegetable

Local people use young

inflorescences as food and vegetable

Local people use young

inflorescences as food and vegetable

Local people use young

inflorescences as food and vegetable

Z. zerumbet (L.) Roscoe ex

inflorescences as food and vegetable

Whole plant is used as

ornamental

Whole plant is used as

ornamental

Rhizomes used for

stomachache

Rhizomes used for

stomachache

Whole plant is used Rhizomes and leaves

as power magic used for stomachache

Rhizome

used to

makeperfume

Rhizomes and young

leaves used for

stomachache

Young leaves used

for stomachache

Whole plant and leaves

used as ornamental

Whole plant used as

ornamental

Whole plant used as

ornamental

Whole plant used as

ornamental

Whole plant used as

ornamental

Whole plant used as

ornamental

Whole plant used as

ornamental

Whole plant used as

ornamental

Whole plant used as

ornamental

Whole plant used as

ornamental

Whole plant used as

ornamental
Whole plant used as

power magic

Whole plant used as

power magic

Whole plant used as

power magic

Rhizomes used as

power magic

Rhizomes used as

power magic

Rhizomes used as

power magic

Rhizomes used as

power magic

Rhizomes used as

power magic

Rhizomes used as

power magic stomachache

Rhizomes used for

stomachache

Rhizomes used for

stomachache

hizomes used for

stomachache

Rhizomes used for

stomachache

Rhizomes used for

stomachache

Rhizomes used for

stomachache

Rhizomes used for

stomachache

Rhizomes used for

stomachache

Rhizomes used for

stomachache

Rhizomes used for

stomachache 


\section{Traditional uses of Zingiberaceae in Udorn Thani Province}

During the surveys, it was found that the villagers from Udorn Thani Province used many species of the family Zingiberaceae as food, spice, medicine, in rituals, as cosmetics, for perfume and as ornamentation (Table 2 and Figures 3-4).

Food. As many as 20 ginger family species were used as local food. The parts of the plants were young inflorescences eaten as vegetables (15 species), rhizomes eaten as vegetables (seven species), young leaves eaten as vegetables (three species), young pseudostems eaten as vegetables (three species), leaves eaten as vegetables (two species) and roots (two species) as the most frequently used for food. Alpinia conchigera, A. galanga, A. siamensis, Boesenbergia rotunda, Curcuma angustifolia, $C$. singularis, and Zingiber officinale are famous for being used in local food.

Spice. The rhizomes of Alpinia conchigera, A. galanga and $A$. siamensis and storage roots of Boesenbergia rotunda were used as spices in local food.

Ornamentals. The second most common use found in this study, with 21 zingiberaceous plants, was ornamental plants. The parts used were reported as whole plants, inflorescences and leaves as the most frequently used for ornamental plants. Hedychium coronarium was the most cultivated species as an ornamental in home gardens.

Cosmetics. The villagers used the rhizome of Curcuma longa as a cosmetic.

Perfume. The rhizomes of Kaempferia marginata or "Toob-Moob" (local name) were used as a local perfume that is called "Nam Hom Udorn Toob Moob".

Ritual plants. There were 18 species of Zingiberaceae that were used as ritual plants. The parts of the plant most frequently used were whole plants (seven species), rhizomes (six species) and inflorescences (five species). Hedychiumcoronarium is commonly used.

Medicine. During the survey of specimens and interviews about the uses of Zingiberaceae from villagers, it was found that 33 species were used as medicine. The parts of the plant most frequently used were rhizomes (33 species), fruits (four species), young leaves (two species), roots (one species) and leaves (one species).

In this study, we documented three tribes, nine genera and 46 species of family Zingiberaceae in Udorn Thani Province. This finding is higher than the study by Saensouk and Saensouk (2020) who reported 18 species of family Zingiberaceae in Phu Phra Bat Historical Park, Ban Phue District, Udorn Thani Province. Curcuma and Kaempferia were the most diverse genera with nine species and eight species, respectively. Zingiber was the third most diverse genus with seven species.

The flowering period was found between March and September, which is similar to a previous study by Saensouk et al. (2016). The fruiting period was found between March and October, which is similar to a previous study by Saensouk et al. (2016). Zingiberaceae in this area was found frequently in five ecology types: cultivated in home gardens (20 species), deciduous dipterocarp forest (19 species), mixed deciduous forest (14 species), dry evergreen forest (six species) and river basin (one species). The results is in accordance with the report of Saensouk et al. (2016).

Kaempferia udonensis and Boesenbergia baimaii were found in a new locality, which differs from previous studies by Phokham et al. (2013) and Saensouk and Larsen (2001). Kaempferia picheansoonthonii and $K$. udonensis were found only in Udorn Thani Province, which is consistent with Phokham et al. (2013). The data from the review references reported four endemic species (Boesenbergia baimaii, B. isanensis, Kaempferia picheansoonthonii and K. udonensis), which agrees with Saensouk and Jenjittikul (2001), Saensouk and Larsen (2001), Phokham et al. (2013), Saensouk and Saensouk (2020), Saensouk et al. (2016) and Wahidah et al. (2020).

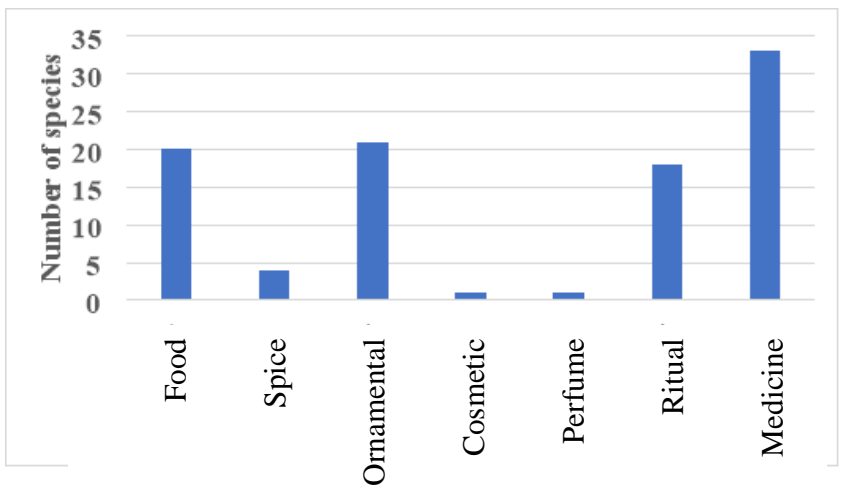

Figure 3. Traditional uses of Zingiberaceae in Udorn Thani Province, Indonesia

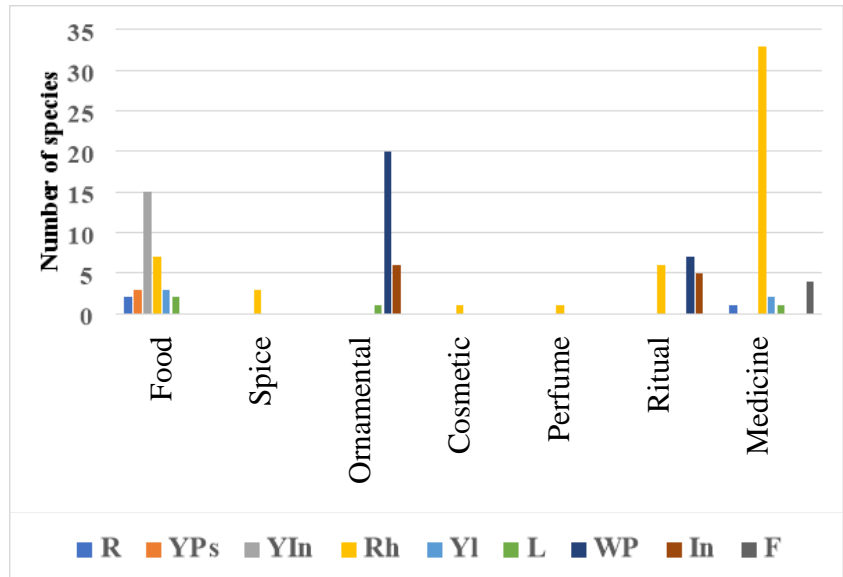

Figure 4. Parts of plant and their main uses for Zingiberaceae in Udorn Thani Province, Indonesia. Notes: Rh: rhizome, YPs: young pseudostem, YIn: young inflorescence, Yl: young leaf, WP: whole plant, L: leaf, R: root, In: inflorescence, F: fruit) 

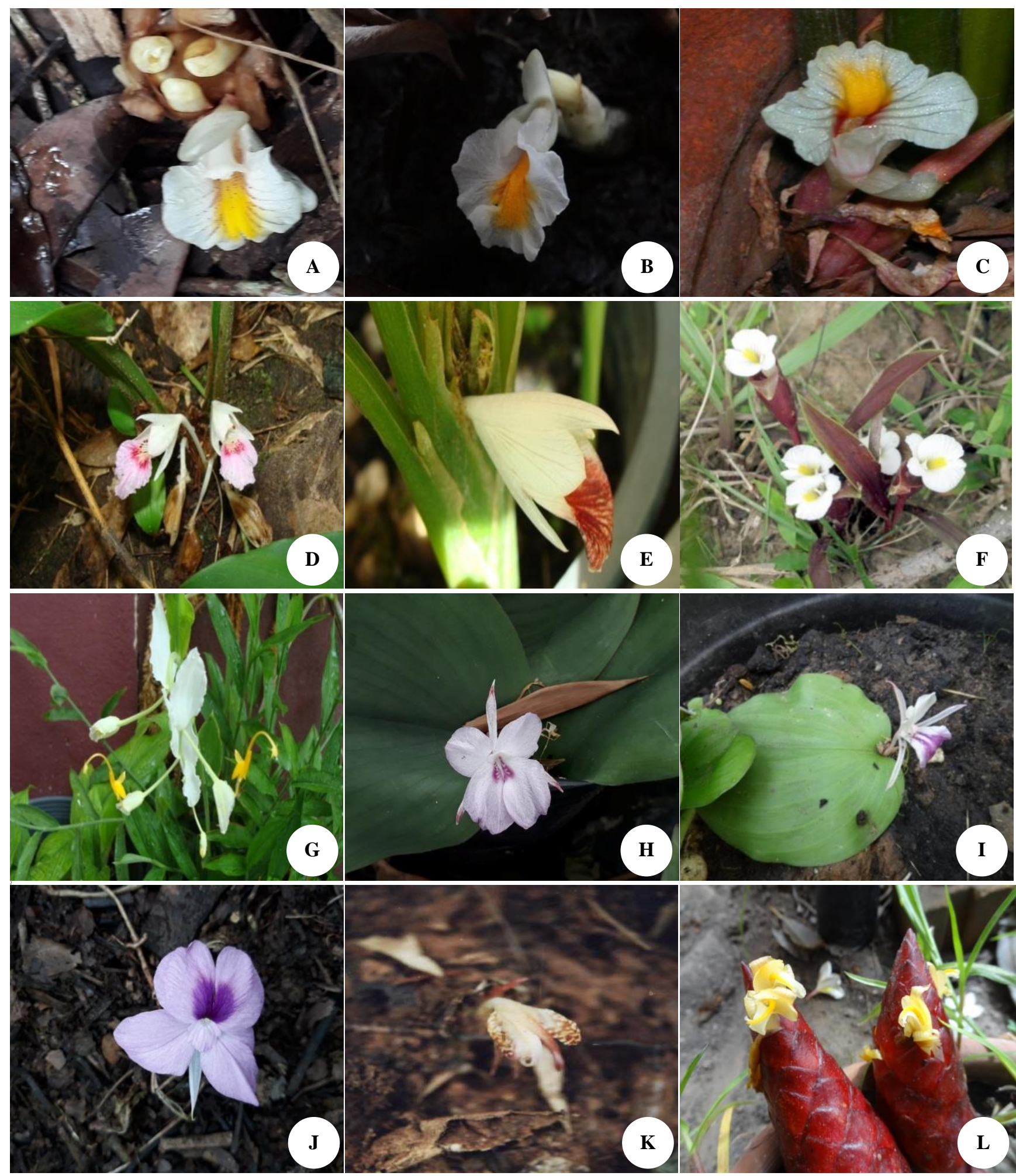

Figure 2. Rare and endemic species of Zingiberaceae in Udorn Thani Province, Thailand. A. Amomum villosum var. xantoides, B. A. trilobum, C. A. wandokthong, D. Boesenbergia baimaii, E. B.isanensis, F. Curcuma campanulatus, G. Globba siamensis, H. Kaempferia picheansoonthonii, I. K. siamensis, J. K. udonensis, K. Zingiber mekongense and L. Z. thorelii 
The conservation status of the family Zingiberaceae in Udorn Thani Province (Table 1) is inline with the report of the IUCN (2021), i.e., least concern or LC (Amomum schmidtii, A. repoense, A. villosum var. xantoides, A. trilobum, Boesenbergia rotunda, Curcuma alismatifolia and Zingiber thorelii), data deficient or DD (Amomum uliginosum) and endangered or E (Globba laeta and $G$. siamensis). Rare species and endemic to Thailand were found, which agrees with several botanists who reported many endemic species, such as Boesenbergia baimaii (Sensouk and Larsen 2001), B. isanensis (Saensouk \& Saensouk 2020), Kaempferia picheansoonthonii (Phokham et al. 2013) and K. udonensis (Phokham et al. 2013).

It was revealed that the uses of many Zingiberaceae species by villagers in Udorn Thani Province were most frequently as medicine, food, ornamentals, in rituals, as spices, perfume and cosmetics, which is consistent with the studies of Sirirugsa (1998), Saensouk et al. (2016), Koga et al. (2016), Pholhiamhan et al. (2018), Saensouk and Saensouk (2019), Phumthum and Balslev (2020), Wahidah et al. (2020) and Pham et al. (2021). The rhizomes, roots, pseudostems, young inflorescences, inflorescences, young leaves, leaves and fruits are the parts of the plant used, which is consistent with the studies of Sirirugsa (1998), Yob et al. (2011), Saensouk et al. (2016), Koga et al. (2016), Pholhiamhan et al. (2018), Saensouk and Saensouk (2019), Phumthum and Balslev (2020), Wahidah et al. (2020) and Pham et al. (2021).

In conclusion, the diversity, phenology data, distribution information, ecology data, conservation status report and traditional uses of the Zingiberaceae in Udorn Thani Province, Thailand between January and December 2020 were determined in this study. Three tribes (Alpinieae, Globbeae and Zingibereae), nine genera with 46 species of Zingiberaceae were identified during the botanical survey. Curcuma, Kempferia, Zingiber, Alpinia, Amomum, Globba and Boesenbergia were the most diverse genera with nine, eight, seven, seven, six, five and three species, respectively. While the genus Etlingera and Hedychium were the least diverse genera with one species each. Keys to tribes, genera and species of family Zingiberaceae in this province were constructed based on the morphological characteristics. Phenological data, including flowering period and fruiting period, were presented between May and October. The flowering period was found between March and September, while the fruiting period was found between May and October. Zingiberaceae was found with the greatest frequency for five ecology types: cultivated in home gardens, deciduous dipterocarp forest, mixed deciduous forest, dry evergreen forest and river basin. Four species-Boesenbergia baimaii, B. isanensis, Kaempferia picheansoonthonii and $K$. udonensis, were found to be endemic species. The conservation status during the observation and collection of specimens can be divided into 26 common species in the research area, 12 rare species in the research area, one rare species in Thailand and five rare species in the world. It was revealed that the traditional uses of many Zingiberaceae species from villagers in Udorn Thani Province were used for medicine, food, as ornamentals, in rituals, as spices, for perfume and cosmetics. Rhizomes, roots, pseudostems, young inflorescences, inflorescences, young leaves, leaves and fruits were the parts of the plants used.

\section{ACKNOWLEDGEMENTS}

This research was financially supported by Mahasarakham University. We would like to thank the Walai Rukhavej Botanical Research Institute and the Faculty of Science, Mahasarakham University for their facilities during the research. I would like to thanks Dr. Jolyon Dodgson (a native English speaker from UK) for language editing and suggestions to improve the manuscript.

\section{REFERENCES}

Ayati Z, Ramezani M, Amiri SM, Moghadam TA, Rahimi H, Abdollahzade A, Sahebkar A, Emami AS. 2019. Ethnobotany, phytochemistry and traditional uses of Curcuma spp. and pharmacological profile of two important species $(C$. longa and $C$. zedoaria): a review. Curr Pharm Des 25 (8): 871-935. DOI: 10.2174/1381612825666190402163940.

Chumroenphat T, Somboonwatthanakul I, Saensouk S, Siriamornpun S. 2019. The diversity of biologically active compounds in the rhizomes of recently discovered Zingiberaceae plants native to North-Eastern Thailand. Pharmacog J 11 (5): 1014-1022. DOI: 10.5530/pj.2019.11.160.

Chumroenphat T, Somboonwatthanakul I, Saensouk S, Siriamornpun S. 2021. Changes in curcuminoids and chemical components of turmeric (Curcuma longa L.) under freeze-drying and low-temperature drying methods. Food Chem 339: 128121. DOI: 10.1016/j.foodchem.2020.128121.

IUCN. 2021. Version $2013.2 \mathrm{http} / / / \mathrm{www}$.iucnredlist.org/

Leong-Škorničková J, Trần HĐ, Nguyê̂́n Quốc Bình, Kristýna Hlavatá, Lưu Hồng Trường, Nguyễn Quốc Đạt, Nguyễn Thành Trung, Newman M. 2019. The identity of Amomum trilobum and Amomum unifolium (Zingiberaceae: Alpinioideae), and description of four new related species from Vietnam. Phytotaxa 401 (3): 149-165. DOI: $10.12705 / 671.2$

Koga YA, Beltrame LF, Pereira VA. 2016. Several aspects of Zingiber zerumbet: a review. Rev Bras Farmacog 26: 385-39. DOI: 10.1016/j.bjp.2016.01.006.

Pham KN, Nguyen TH, Nguyen BQ. 2021. A review on the ethnomedicinal uses, phytochemistry and pharmacology of plant species belonging to Kaempferia L. genus (Zingiberaceae). Pharm Sci Asia 48 (1): 1-24. DOI: 10.29090/psa.2021.01.19.070.

Phokham B, Wongsuwan P, Picheansoonthon C. 2013. Three new species of Kaempferia (Zingiberaceae) from Thailand and Laos. J Jpn Bot 88 (5): 297-308.

Pholhiamhan R, Saensouk S, Saensouk P. 2018. Ethnobotany of Phu Thai ethnic group in Nakhon Phanom Province, Thailand. Walailak J Sci Technol 15 (10): 679-699. DOI: 10.48048/wjst.2018.3737.

Phumthum M, Balslev H. 2020. Using ICPC-2 standard to identify Thai Zingiberaceae of pharmacological interest. Plants 9 (7): 906. DOI: 10.3390/plants9070906.

Saensouk S, Jenjittikul T. 2001. Kaempferia grandifolia sp. nov. (Zingiberaceae) a new species from Thailand. Nordic J Bot 21 (2): 139-142. DOI: 10.1111/j.1756-1051.2001.tb01349.x.

Saensouk S, Larsen K. 2001. Boesenbergia baimaii, a new species of Zingiberaceae from Thailand. Nordic J Bot 21 (6): 595-597. DOI: 10.1111/j.1756-1051.2001.tb00818.x.

Saensouk S, Saensouk P. 2014. Elettariopsis biphylla, a new species of Zingiberaceae from Thailand. Phytotaxa 159 (1): 23-25. DOI: 10.11646/phytotaxa.159.1.4. 
Saensouk S, Saensouk P. 2019. Kaempferia mahasarakhamensis sp. nov. (Zingiberaceae), a new species from Northeastern Thailand. Taiwania 64 (1): 39-42. DOI: 10.6165/tai.2019.64.39.

Saensouk S, Saensouk P. 2020. Boesenbergia isanensis (Zingiberaceae), a new species from Thailand. J Jpn Bot 95 (2): 65-68.

Saensouk S, Boonma T, Saensouk P. 2021. Six new species and a new record of Curcuma L. (Zingiberaceae) from Thailand. Biodiversitas 22 (4): 1658-1685. DOI: 10.13057/biodiv/d220410.

Saensouk S, Saensouk P, Pasorn P, Chantaranothai P. 2016. Diversity, traditional uses and new record of Zingiberaceae in Nam Nao National Park, Petchabun Province, Thailand. Agric Nat Resour 50: 445-453. DOI: 0.1016/j.anres.2016.08.002.

Sirirugsa P. 1998. Thai Zingiberaceae: species diversity and their uses. International Conference on Biodiversity and Bioresources:
Conservation and Utilization, 23-27 November 1997, Phuket, Thailand.

Udorn Thani Province. 2017. https://www.google.co.th/maps/place/

Wahidah FB, Hayati N, Khusna NU, Rahmani DPT, Khasanah R, Kamal I, Husain F, Setiawan IA. 2020. The ethnobotany of Zingiberaceae as the traditional medicine ingredients utilized by Colo Muria mountain villagers, Central Java. J Phys: Conf Ser 1796 (1): 012113. DOI: 10.1088/1742-6596/1796/1/012113.

Yob NJ, Jofrry M, Affandi MMR, Teh LK, Salleh MZ, Zakaria ZA, 2011. Zingiber zerumbet (L.) Smith: a review of its ethnomedicinal, chemical, and pharmacological uses. Evid Based Compl Altern Med. DOI: $10.1155 / 2011 / 5432$. 